\title{
Improving Cell Phone Awareness by Using Calendar Information
}

\author{
Ashraf Khalil and Kay Connelly \\ Department of Computer Science, Indiana University, \\ Bloomington, IN, 47405 \\ \{akhalil, connelly\}@cs.indiana.edu
}

\begin{abstract}
The many benefits that cell phones provide are at times overshadowed by the problems they create, as when one person's cell phone disrupts a group activity, such as a class, meeting or movie. Cell phone interruption is only highlighted by the ever increasing number of mobile devices we carry. Many tools and techniques have been proposed in order to minimize interruption caused by mobile devices. In the current study, we use calendar information to infer users' activity and to automatically configure cell phones accordingly. Our in-situ experiment uses PDAs that run a cell phone simulator to examine the feasibility and design factors of such a solution. Our results show that both structured activities and appropriate cell phone configuration can be predicted with high accuracy using the calendar information. The results also show consistent mapping of activities to configuration for each individual. However there was a poor consistency of mapping activity to configuration across different participants. We discuss the results in relation to inaccuracy, spontaneous activities, and user reactions.
\end{abstract}

\section{Introduction}

With the increasing number of mobile devices that seek users' attention, it is essential to minimize interruptions and distractions caused to the users and the surrounding environment. Garlan et. al. notes that human attention is becoming the most precious and scarce resource, considerably more so than computational power [1]. Cell phones, with all the services they provide such as phone calls, reminders, text and instant messages, are the prime example of mobile devices that demand constant cognitive attention from the user and also serve as a frequent source of interruption and distraction. Cell phones are currently the most ubiquitous communication device the world over [2]. The tremendous growth of cell phones' usage and their location-free nature have helped to establish a new social order. This social order has been described as a shift from Place-to-Place communication to Person-to-Person communication [3].

The benefits offered by cell phones, such as flexibility and accessibility, seem to inevitably come with the cost of increased interruption and interaction demands. Interruption caused by inappropriate notification such as ringing in a meeting can cause inconvenience, disruption and embarrassment for the owner. The effect of interruptions has been shown to be disruptive to task performance even when the 
interruption is ignored [4]. Even worse, interruption may also lead to an increased level of stress and errors $[5,6]$. Interruption is not limited to the owner of the cell phone only but extends to the surrounding environment as well. Kern et al [7] have introduced and validated a model for interruptibility wherein they distinguish between interruption to user's environment "social interruptability" and interruption to the user him or herself "personal interruptability". The cell phone's social interruptibility is further confirmed by studies that show most people consider the use of cell phones in public places to be annoying [8, 9]. Bautsch, et. al [10] found that most people think there should be etiquette guidelines created for public mobile phone use.

All the problems mentioned above are usually caused by the static nature of cell phone configurations and their inability to automatically change their setting according to the context of the surrounding environment. This, in turn, creates a mismatch between a phone's setting and the context of the space it occupies. Many people change their cell phone setting every time they are in a new context. This solution is both inconvenient and inadequate since in many cases the user forgets to change the setting. Other people just keep their cell phone in silent mode, but that results in their missing many important calls.

In this paper, we present an approach that aims to improve the awareness of cell phones by using information from the calendar book, which already exists in most cell phones and all current smart phones. The information in the calendar book is used to determine the most suitable configuration for the cell phone. In order to examine the validity and effectiveness of this solution, many questions need to be explored first. Its real value greatly depends on the accuracy of the predicted context based on the scheduled activities. Given the inevitable fact that people's actions do not always mirror their intentions, scheduling events and activities does not necessarily ensure attendance.

With this in mind, the accuracy of the information provided by the calendar must be carefully considered, along with the tendency of users to carry out their plans as written in the calendar. A related question to be asked concerns the effect of spontaneous and unscheduled activities on the predictability of calendar-based configuration. Further, can users predict the best configuration for specific activities? Is there consistent mapping between context and configuration? Given that people's sense of control decreases as a cell phone's autonomous capabilities increases [11], and given the personal connection people feel toward their cell phones, would people welcome the idea of more aware and autonomous cell phones? How much control are users willing to give up in exchange for the convenience offered by the system? Finally, how can we account for the differences in people's perception of the appropriateness of the same level of interruption?

To answer the above questions, we have conducted an in situ experiment in a dynamic campus setting. During the experiment, participants were asked to fill in their calendar information regularly in the PDA we supplied. Every PDA ran an application designed to simulate a smart cell phone. The application simulated phone calls at random times during the day, prompting participants to evaluate the appropriateness of the configuration and to specify their current activity and location. The application acquired and stored evaluation data from participants during the study. More data was collected through end-of-study interviews to examine the overall evaluation of the calendar-based solution. 
Section 2 examines the existing solutions to this problem at hand. We describe the strengths of the calendar book as a solution in Section 3, and give the details of our in-situ user study in Section 4. Section 5 documents our results, including the accuracy of calendar information, the consistency of the mappings between context and configuration in our user population participants', and evaluations of calendarbased configuration. We conclude our findings in Section 6.

\section{Calendar as a Context Provider}

All current smart phones and most other regular cell phones come equipped with a calendar book. The calendar book usually serves as a personal organizer and is a valuable resource for organizing daily activities and schedules. Naturally, calendar information provides very important and reliable cues about the availability, location, and surrounding environment of the user. For example, if the calendar has a meeting appointment from $1 \mathrm{pm}$ to $2 \mathrm{pm}$ we know with a high degree of probability that the user is unavailable and he is in a place with at least one other person. Such information indicates that any incoming interruption should be kept minimal and only the most urgent ones should be allowed to go though with a very discreet notification mechanism. Such cues are available for free and we predict they are usually accurate.

Calendars provide simple and inexpensive contextual information. By inexpensive, we mean that no sensors or computations are needed to infer the contextual information. Furthermore we predict this information to be highly accurate since in most cases the user fills in the entries that are of high importance and that she intends to attend. This information can be used by cell phones in order to dynamically and automatically change their configuration, or settings, in a way that received calls are least disruptive to both the user and the surrounding environment.

It remains unclear, however, to what extent cell phone calendars are actually used. We have not found any studies that examine the usage pattern of a cell phone's calendar, although many studies have been conducted to examine the mobile Personal Information Management's (PIM) usage behavior, task management, and efficiency of information retrieval [12]. PIM is an essential set of tools that exists in almost all personal digital assistants (PDAs) as well as many smart phones and includes a calendar application in addition to task list, contact, and memo applications. In a recent study we conducted involving 20 cell phone users, we found that a cell phone's calendar is rarely used, or when it is used, it serves as a reminder rather than as a scheduler. However, we expect this to change in the near future as regular cell phones converge into smart phones that include many PDA-like capabilities. Smart phones usually offer better user interface and communication capabilities. Enhanced interaction capabilities such as bigger touch-screen displays and a QWERTY keyboard provide for easier user input and enable users to make better use of PIM applications, including the calendar. Moreover, the enhanced communication capabilities such as Bluetooth and infrared enable users to synchronize their calendar information and other data with their PC. Thus, even if users utilize other electronic calendar devices, the information can easily be transferred to and used by their smart phone. 


\section{Related Work}

Calendar information has long been used as a valuable resource for information in several research projects. The Coordinate system, for example, uses the calendar information and previous computer activities to predict the availability of a person on a particular computing device [13]. MyVine system uses calendar information in addition to many other cues, such as a speech detection sensor, to model a person's availability for communication [14]. The Ambush system extends the calendar via a Bayesian approach to predict the likelihood of one's attendance at the event listed in one's calendar [15]. The context-aware Office Assistant uses a person's calendar to inform a personal agent of available meeting times for visitors at the person's office door [16].

Many approaches have been developed aiming to minimize interruptions caused by mobile devices. One approach is to empower the caller to make better decisions about the appropriateness of the call before making it by providing him information about the receiver's context [17-19]. The Calls.calm system uses the web to activate an interaction webpage that provides the caller with a set of available communication channels as well as information about the receiver's current situation and leaves it up to the caller to make an educated choice [17]. Milewski and Smith used the address book to display dynamic information about the recipient's availability and whereabouts [19]. The solution applies the same concepts of "Buddy list", used in instant messengers. This approach, however, raises many privacy concerns that may prevent the receivers from publishing useful information about their context. Also the approach does not address the question of what type of contextual information provides the best cues about the receiver's availability.

Another approach is to empower cell phone owners by improving the capabilities and awareness of cell phones. Quiet Calls is a system that enables users to have a private conversation in a public place by using a quiet mode of communication such as voice mail and prerecorded messages [20]. Such a system decreases social interruption but does not affect personal interruption since the user is still expected to receive the call and act upon it. SenSay is a system that uses input from different sensors such as accelerometers, light detectors, and microphones to capture the context of the user [21]. The context is then used to adjust the modality of cell phone configuration (i.e. vibration, ringer). Schmidt et. al. have introduced an adaptive cell phone that changes its profile automatically based on the recognized context [22]. The phone chooses to ring, vibrate, adjust the ring volume, or keep silent depending on whether the phone is on a table, in a suitcase, outside, or in hand. Solutions which acquire context information through augmented sensors are somewhat expensive in terms of the computational needs of inferring the context information given the scarce resources of cell phones. Moreover, usability studies have yet to be conducted to study the effect of inaccurate context prediction on users, as well as the issue of how much control users are willing to give up in exchange for convenience. 


\section{Experiment}

The main goal of the experiment was to assess the likely value of the calendar-based automatic cell phone configuration approach and the various factors affecting it. We examined the accuracy of calendar information, configuration predictability, and the consistency of mapping activities to configurations for individuals and across different individuals. We examined whether automatic cell phone configuration, based on the user's calendar information, improves the overall user experience. Finally, we investigated the approach to automatic configuration and whether it should be passive, where users are aware of the change and have more control over it, or active, where the change is made without any notification and the user has less control over it.

\subsection{Preliminary Survey}

The experiment was conducted in two stages. Preliminary data was collected using a survey in the first stage to help us better design the main part of the experiment. The benefit of a two-stage experimental approach in the context of interactive and ubiquitous systems was argued by Antifakos et al. [23]. The goal of the survey is to investigate how people categorize their daily activities as well as the variation of this categorization across different groups. The data was gathered by an online survey. We had a total of 72 participants divided among graduate students, undergraduates, professors and staff. The participants were distributed among 7 different majors or areas of study. The survey results show that the participants tend to do very similar activities irrespective of their major or occupation. However, we found that the frequencies of activities are different among different groups. We used the list of collected activities to categorize them according to the most suitable cell phone configuration corresponding to that activity.

\subsection{Method}

Design: The study consists of a context-aware cell phone configuration application. The application simulates a cell phone that changes its configuration (loud ring, quiet ring, vibrate, on, off) depending on the context of its owner. The context is derived from the calendar book. During the study, the participant carries a Palm PDA that runs the application, and during the day she receives simulated phone calls at random times (Figure1). According to the context of the participant, the application notifies the user differently about the received call. The cell phone configuration can be in any of four different states: Loud, Quiet, Off, and Normal. In the loud state, the phone rings loudly when a call is received, while it vibrates in the Quiet state. Normal state is the default state that takes whichever configuration has been set up by the owner. In the Off state, the phone is off, and if a call is received then a voice mail message will be generated the next time the phone is in any other state. Moreover, if the participant misses a phone call, he will be notified of that missed call the next time he answers a phone call. The four different states were identified from the online preliminary survey mentioned in the previous section. After receiving the notification of either an incoming call, a missed call or a voice mail message, the participant is asked whether 
the configuration of the cell phone, reflected by the notification mechanism, is appropriate or not. If the answer was inappropriate, then she is asked to select the most appropriate configuration. After that, the participant is asked to select his location and activity.

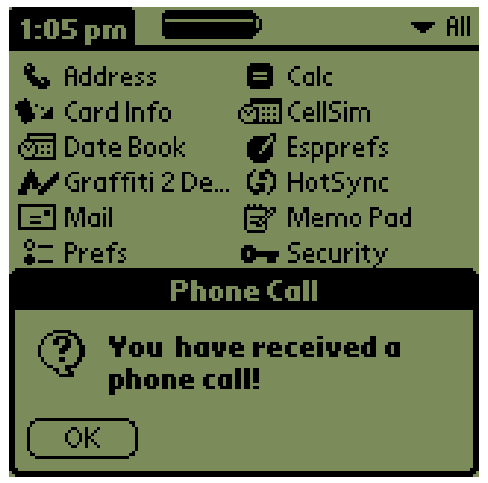

(a)

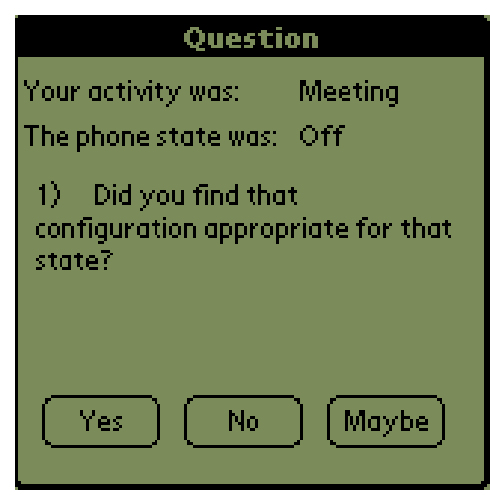

(b)

Fig. 1. Figure (a) shows the notification message that appears once a call is received. Figure (b) shows the question asked once the user presses the "OK" button on figure (a).

We chose to use a cell phone simulator that is running on a Palm PDA instead of using a real cell phone because the PDA provided us with more programming flexibility and with greater means to collect, store and manage in-situ data. At the same time, the selected PDAs had the same notification capabilities as cell phones, such as ringing, vibrating, LED, and volume control. We were only interested in measuring the appropriateness of the configurations in terms of their social ramifications rather than identifying the callers or any other factors. The simulation provided us with more control over the study, which enabled us to examine only the factor of interest while eliminating others such as caller identity. Moreover, it would have been very expensive and inefficient to provide participants with real cell phones with calling plans for the duration of the study.

Conducting the experiment in a natural setting is a crucial part of our study and for Ubicomp systems in general because of their inherent mobile nature and the fact that, in real life, they interact with users in a natural environment. We have chosen to conduct the experiment in a college environment because of its dynamics and the ease of subject recruitment. Moreover, college campuses have always been on the forefront of adopting new technologies. In fact, Weiser [24] predicted that the compact nature of the campus environment will put it at the forefront of ubiquitous computing.

Duration: The experiment duration was chosen to be 5 working days. This period was selected because most activities are repeated in either daily or weekly intervals. In addition, we conducted the experiment only during the week rather than on the weekends because we were mostly interested in the days when the participants are busy and interactive in a campus environment. In this case, the cost of interruption or 
misconfiguration is rather high for both the user and the surroundings and thus the value of the application is highlighted.

Participants: 11 students both graduate and undergraduate from Indiana University participated in the study. Participants were aged 20-28 and 3 of them were males. All participants reported to have owned cell phones for more than a year and have busy daily schedules with many different activities throughout the day. 10 participants fully completed the study. One participant collected very little data due to a family emergency. This data was not considered in the evaluation process.

Equipments: The study was conducted using Tungsten T3 running Palm OS 5.2 and our cell phone simulator. The devices are equipped with ringing, vibration and volume control capabilities as well as a color display. Each participant was provided with a PDA for the duration of the study.

Procedure: Participants were individually given a brief overview on how to use the PDA, and then they were introduced to the cell phone simulator and how to use it. They were asked to fill in the calendar with their activities at the beginning of every day of the study with all the activities that last at least 15 minutes. Every activity is mapped by the participant to the cell phone state that best fits that activity. The participants were advised to think of the PDA as their own cell phone that is changing its configuration dynamically depending on the context. After finishing with the experimental study, end-of-study interviews were conducted in one-on-one sessions that lasted approximately 40 minutes.

Design Tradeoffs: The fact that participants received simulated phone calls rather than real ones may have introduced some bias in their evaluation of the calls. In order to treat all calls with the same level of importance and factor out personal preferences, we asked participants to think of the calls as received from anonymous callers. With the simulated phone calls, participants still had to deal with the social ramifications of receiving calls in public spaces and with inappropriate alerts that could have been caused by the calls. Also, activity to configuration mapping in most cases should not be affected by the fact that the calls are simulated.

\section{Results and Discussion}

During the study, a total of 340 calls were made, all generated by the simulator. Participants received an average of 30 calls and 4 voice mails. Even though participants missed $31 \%$ of the initial calls, they received reminders about many of the missed calls, and thus they had the chance to evaluate them. Overall participants evaluated $85 \%$ of the calls; the rest were not evaluated due to the fact that the application only stored a partial list of missed calls and the participants were reminded only about the last three missed calls. In addition, in some cases, the Palm device had to be reset during the study, and thus a few stored reminders were lost. 


\subsection{Evaluating Calendar-Based Automatic Cell Phone Configuration}

During the end-of-study interview, all participants reported they were willing to use such an application if their cell phones were equipped with it. Participants were also asked to rate the usefulness of the application on a scale of 1 to 6 , with 1 being the most useful and 6 being annoying. $40 \%$ of the participants rated it 1 (very useful) while the rest rated it 2 (useful). These results are particularly interesting given the fact that $9 \%-13 \%$ of the calls were evaluated as having an inappropriate or inexact configuration. Since these calls were received in a real-life environment, they could have caused frustration or embarrassment for the participants. The fact that evaluation occurs after notification makes the evaluation very accurate and reflects the real feelings of the participants that could not be obtained otherwise. One participant commented that: "I like how it changes state without you having to tell it to. I always forget to turn my cell [off] in class and turn it on after".

Overall, participants rated $87 \%$ of the evaluated calls as having the appropriate configuration and $9 \%$ as having an inappropriate configuration. The rest were evaluated as having an inexact configuration but not inappropriate which was another option. Out of the missed calls that were later evaluated, $36 \%$ were missed unintentionally due to the fact that participants failed to notice the alert, usually due to low volume, and the rest were missed intentionally. We interpreted both intentionally and unintentionally missed calls as having an inappropriate configuration. In most cases participants did not mind missing the calls because they did not want to be interrupted. Only $14 \%$ of the missed calls were evaluated as having an inappropriate configuration. One participant commented that the embarrassment of having the phone ring in the middle of a meeting is worse than missing a phone call. Most of the calls with inappropriate configuration were received when the participants were either in transition between activities or dealing with unplanned activities such as 'on the phone', 'taking a break' or 'having a conversation'.

Even though this approach did not produce perfect accuracy, it did not appear to affect the participants' perception about the usefulness of the applications since any inaccuracy is a predictable one and they have total control over it. People's reaction to inaccuracies and uncertainty in context-aware applications varies from one person to another. However, if Bellotti and Edwards' [25] design principles of intelligibility and accountability are followed, which include the user in the decision making process, then we expect people will adopt context-aware applications. In our case, participants were included in the decision making process by having them initially map different states to configuration rather that using different inference techniques to map them automatically.

Our study also examined the preferred level of interactivity between the user and any potential application that could provide context-aware configuration for cell phones. This question must be dealt with for most of the context-aware applications because of the fact that they are dynamic and proactive [26]. When asked about their interaction preferences in the end-of-study interviews, all participants stated that they would like to be involved in deciding on the configuration for a given context. Most participants wanted to be notified whenever certain configuration transitions occur. However, the level of involvement varies from one participant to another. A detailed discussion of this can be found in [27]. 


\subsection{Accuracy of Calendar Information}

Participants were asked to fill in a more detailed account of their daily activities than they would usually do in real life. We asked them to schedule in advance all the activities that were expected to last more than 15 minutes. The purpose was to gather as much data as we could about the different activities and the way participants choose to configure these activities. Participants filled in 9 different activities on average. The most common activities were meeting, work, homework, watching TV, class, working out and eating. During the study, participants were asked to specify their current activity. Participants accurately predicted $62 \%$ of their activities and inaccurately predicted $29 \%$ of activities. $9 \%$ of participant activities were spontaneous. The relatively low value for accuracy was not unexpected given the fact that it is very hard to predict a detailed account of our daily activities in advance. The calendar is designed to function as an organizer of important, well-structured events and not activities part of one's daily routine. Not surprisingly, the activities that contributed to most of the inaccuracies in calendar predictability were the loosely structured home activities such as "food", "watching TV", "homework" and "relaxing". When such activities were ignored, a much higher accuracy rate of $93 \%$ was obtained. This result highlights the importance of using calendar information as a source of contextual cues with high level of accuracy for structured activities.

\subsection{Mapping Activities to Configuration}

One main goal of the experiment was to examine how people map their activities to different configurations and to check for consistency in the mappings. In order for the configuration to be determined automatically by the cell phone (i.e. inferring it from the description field in the calendar entry) and not as specifically directed by the owner as in our experiment, there needs to be a predictable pattern of mapping from activities to configuration.

Table 1. Activity to configuration mapping by one participant

\begin{tabular}{|c|c|c|c|c|}
\hline Activity & Loud & Normal & Quiet & Off \\
\hline Meeting & 0 & 2 & 6 & 1 \\
\hline Work & 0 & 6 & 5 & 1 \\
\hline errands & 0 & 1 & 0 & 0 \\
\hline Class & 0 & 0 & 3 & 5 \\
\hline Homework & 0 & 10 & 0 & 0 \\
\hline Lunch & 0 & 3 & 0 & 0 \\
\hline Watching TV & 0 & 1 & 0 & 0 \\
\hline Work Out & 0 & 0 & 1 & 1 \\
\hline Walking & 0 & 1 & 0 & 0 \\
\hline Shopping & 2 & 0 & 0 & 0 \\
\hline
\end{tabular}

A typical example of the mapping data that was collected is shown in Table 1 . Upon initial observation, it appears that most activities have a dominant desired 
configuration, but that there exist activities that have 2 or more preferred configurations. Upon closer examination, however, we find that individuals have a predictable desired configuration for $89 \%$ of the activities. For example, the "work" activity in Table 1 shows two different dominant configurations. This particular case was due to the fact that the user happened to configure the "Normal" state to the same setting as the "Quiet" configuration. Thus, the "work" activity for this participant is counted as consistent. Further, configurations other than the dominant ones were usually chosen at the beginning of the study when participants were experimenting with the settings and were not yet sure which was the best fit for a particular activity. This behavior tended to diminish toward the end of the study period. This shows that the mapping process can be easily automated after the initial period where the user is more involved and she is part of the decision making.

The mapping was less consistent across different participants than for each individual participant. Table 2 shows the activity to configuration mapping data for the eight most common activities among participants. Even though certain activities such as "Homework" showed consistent mapping, many other activities were not as consistent. For example, many participants chose to have their cell phone "Off" during class, but others chose the "Quiet" configuration. As a result of this and in order for the automatic configuration to be useful, it should be tuned and customized to specific preferences for each user. However, results from this type of study can be used to choose intelligent default settings for different activities. For example, the default setting for "Work" could be "Quiet" because it is the most popular configuration. The users who desire "Normal" can change this default.

Table 2. Activity to configuration mapping across all participants

\begin{tabular}{|c|c|c|c|c|}
\hline Activity & Loud & Normal & Quiet & Off \\
\hline Meeting & 2 & 25 & 23 & 6 \\
\hline Homework & 8 & 57 & 2 & 0 \\
\hline Class & 1 & 0 & 18 & 29 \\
\hline Food & 1 & 21 & 9 & 0 \\
\hline Work Out & 2 & 2 & 2 & 1 \\
\hline Travel & 24 & 29 & 2 & 0 \\
\hline Watching TV & 3 & 9 & 2 & 0 \\
\hline Work & 0 & 17 & 38 & 4 \\
\hline
\end{tabular}

Kern. et. al obtained very similar results in their study of the differences between personal and social interruptability [7]. Their experiment found that there were differences among people in the way they assess personal and social interruptability and they argue for interruptability estimation systems to better adapt to individual users' preferences. This may well be the case for all other interactive, context-aware applications. Making general conclusions about the desired behavior of context-aware applications within certain contexts is a problematic practice that many researchers have fallen into. In fact this is the same practice that Bellotti and Edwards have warned about, and they have proposed following the guidelines of intelligibility and 
accountability when designing context-aware systems to avoid such problems [28]. We expect that providing the capability for participants to choose their own mapping from activities to the desired configuration has had a substantial effect on the way participants perceive the application as well as on the very positive evaluation of its usefulness.

\section{Conclusion}

Our results suggest that automatic configuration based on calendar information provides both an effective and desirable solution to the interruption problem caused by cell phones. The results show that both structured activities and appropriate configuration can be predicted with high accuracy using the calendar information. The results also show consistent mapping of activities to configuration for each individual. However there was a poor consistency pf mapping activity to configuration across different participants. The results show that people are willing to accept a certain level of inaccuracy which comes as a side effect of any context-aware application in exchange for good services and convenience.

Calendar information does not provide accurate context all of the time, and even if the context is predicted accurately, the desired configuration for a certain context is not always the same and there are many factors that might affect it. However, even with an inaccuracy rate of $9-13 \%$, participants still liked this solution and said they are willing to adopt it in real life. We believe this inaccuracy rate can be greatly reduced if reinforcement learning tools were used over a longer period of time. Moreover, the fact that people use the calendar for important activities and appointments and not to record a detailed account of their daily activities as they were asked to do during the study, is expected to increase the accuracy of context predictability and the consistency of activity to configuration mapping.

The fact that mobile phone calendar usage might not be very common due to the very limited inputting capabilities should not undermine the importance of our results. Mobile phones are developing at a very fast pace and smartphones are gaining more and more popularity. Smartphones offer more interaction capabilities, coupling phone capabilities with the functionalities of a PDA, and short-range wireless connectivity such as Bluetooth. The PDA functionalities of the smartphones are expected to drastically increase the use of the mobile calendar application as well as other PIM applications. Moreover, the short-range connectivity is expected to further contribute to the popularity of the mobile calendar due to the fact that people can use their computers to fill in the entries and use the wireless connection to synchronize with their smartphones.

The goal of future work should be to study the relationship between the caller and the receiver and its effect on how people evaluate the appropriateness of different phone configurations given different roles of the caller. In addition, we are planning to do the same experiment with real cell phones in order to validate the results and avoid any biases that could be introduced by the simulation. It is also important to examine the preferred direction of error in a context-aware configuration. The error of such an application can be of two sorts: fewer missed calls but higher probability of inappropriate interruption or fewer inappropriate interruptions but more probability of 
missed calls. In our experiment, most participants were not annoyed by missing calls since the caller was assumed to be anonymous. That the calls were not real may have contributed to this factor; however, this may not be the case in real life. We would like to investigate whether it is more important for people not to be interrupted inappropriately or not to miss certain calls. It is also important to study the effect of using real mobile calendar information as opposed to using a structured list of activities, as was the case in our study. A real strength of our study, however, is that it provided valuable insight regarding activity-to-configuration mapping and its consistency, as well as validity and usefulness of the calendar-based approach to decrease cell phone interruptions. We thereby hope to influence the design of future applications' aims regarding context-aware configuration.

\section{Acknowledgments}

This work was supported in part by NSF (EIA-0202048) and by a grant from the Lilly Endowment. We would also like to thank the subjects who provided us with valuable data and feedback.

\section{References}

1. Garlan, D., et al., Project Aura: Toward Distraction-Free Pervasive Computing. IEEE Pervasive Computing, 2002(April-June).

2. REUTERS, Mobile phone users double since 2000. Dec, 2004.

3. Wellman, B., Physical Place and Cyber Place: The Rise of Personalized Networking. International Journal of Urban and Regional Research, 2001. 25(2): p. 227-52.

4. Cutrell, E., M. Czerwinski, and E. Horvitz. Notification, Disruption, and Memory: Effects of Messaging Interruptions on Memory and Performance. In Proceedings of Interact 2001. 2001. Tokyo, Japan.

5. Eyrolle, H. and J. Cellier, The effects of interruptions in work activity: Field and laboratory results. Applied Ergonomics, 2000. 31: p. 537-543.

6. Study: All cell phones distract drivers. August 16, 2001, CNN.

7. Kern, N., et al. A model for human interruptability: experimental evaluation and automatic estimation from wearable sensors. In Proceedings of IEEE International Symposium on Wearable Computers. 2004.

8. Monk, A., et al., Why are Mobile Phones Annoying? Behaviour and Information Technolog, 2004. 31(1): p. 33-41.

9. Lasen, A., A comparative Study of Mobile Phone Use in London, Madrid and Paris. 2002

10. Bautsch, H., et al., An investigation of mobile phone use: a socio-technical approach. 2001

11. Barkhuus, L. and A.K. Dey. Is context-aware computing taking control away from the user? Three levels of interactivity examined. In Proceedings of UBICOMP 2003, 5th International Symposium on Ubiquitous Computing. 2003.

12. Bellotti, V., et al. What a to-do: Studies of task management towards the design of a personal task list manager. In Proceedings of CHI 2004. 2004: ACM Press (2004).

13. Horvitz, E., et al. Coordinate: Probabilistic Forecasting of Presence and Availability. In Proceedings of National Conference on Uncertainty and Artificial Intelligence (UAI 2002). 2002. 
14. Fogarty, J., J. Lai, and J. Christensen, Presence versus Availability: The Design and Evaluation of a Context-Aware Communication Client. International Journal of HumanComputer Studies (IJHCS), 2004. 61(3): p. 299-317.

15. Mynatt, E. and J. Tullio. Inferring Calendar Event Attendance. In Proceedings of the International Conference on Intelligent User Interfaces (IUI 2001). 2001.

16. Yan, H. and T. Selker. Context-aware office assistant. In Proceedings of International Conference on Intelligent User Interfaces. 2000. New Orleans, LA.

17. Pedersen, E.R. Calls.calm: Enabling Caller and Callee to Collaborate. In Proceedings of CHI 2001. 2001.

18. TANG, J.C., et al. ConNexus to awarenex: extending awareness to mobile users. In Proceedings of CHI 2001. 2001.

19. Milewshi, A.E. and T.M. Smith. Providing Presence Cues to Telephone Users. In Proceedings of CSCW 2000. 2000. Philadelphia, PA.

20. Nelson, L., S. Bly, and T. Sokoler. Quiet Calls: Talking Silently on Mobile Phones. In Proceedings of CHI 2001 Conference on Human Factors in Computing Systems. 2001.

21. Siewiorek, D., et al. SenSay: A Context-Aware Mobile Phone. In Proceedings of IEEE International Symposium on Wearable Computers (ISWC). 2003. New York, NY.

22. Schmidt, A., et al. Advanced interaction in context. In Proceedings of First International Symposium on Handheld and Ubiquitous Computing (HUC'99). 1999. Karlsruhe, Germany.

23. Antifakos, S., A. Schwaninger, and B. Schiele. Evaluating the Effects of Displaying Uncertainty in Context-Aware Applications. In Proceedings of Ubicomp'04. 6th International Conference on Ubiquitous Computing. 2004. Nottingham, UK.

24. Weiser, M., The Future of Ubiquitous Computing on Campus. Communications of ACM, 1996. 41(1): p. 41-42.

25. Bellotti, V. and K. Edwards, Intelligibility and accountability: Human considerations in context-aware systems. Human-Computer Interaction, 2001. 16: p. 193-212.

26. Chen, G. and D. Kotz, A survey of context-aware mobile computing research. 2000, Department of Computer Science, Darthmouth CollegeTR2000-381.

27. Khalil, A. and K. Connelly. Context-aware Configuration: A study on improving cell phone awareness. In Proceedings of Context 05. July 2005. Paris, France.

28. Bellotti, V. and W.K. Edwards, Intelligibility and accountability: human considerations in context aware systems. Human Computer Interaction, 2001. 16(2-4): p. 193-212.

29. Dourish, P. and V. Bellotti. Awareness and Coordination in Shared Workspaces. In Proceedings of ACM Conference on Computer Supported Cooperative Work (CSCW). 1992. 